\title{
Frecuencia y algunos factores de riesgo de mortalidad en el estado de Hidalgo, México, por defectos de cierre del tubo neural
}

\author{
Sergio Muñoz-Juárez, M en $C{ }_{1}{ }^{(1)}$ Humberto Vargas-Flores, MC, MSP, ${ }^{(2)}$ Bernardo Hernández-Prado, PhD, (3) \\ 0 lga López-Ríos, PhD, ${ }^{(4)}$ Rosa María 0 rtiz-Espinosa, M en $C .{ }^{(5)}$
}

Muñoz-Juárez S,Vargas-Flores H, Hernández-Prado B, López-Ríos O, Ortiz-Espinosa R.

Frecuencia y algunos factores de riesgo

de mortalidad en el estado de Hidalgo,

México, por defectos de cierre del tubo neural.

Salud Publica Mex 2002;44:422-430.

El texto completo en inglés de este artículo está disponible en: http://www.insp.mx/salud/index.html

\section{Resumen}

Objetivo. Calcular el riesgo de muerte fetal secundaria a defectos del cierre del tubo neural y estimar factores asociados con este tipo de muertes en el estado de Hidalgo. Material y métodos. La información analizada en el año 2000 fue obtenida de los certificados de muerte fetal del perio do 1990-1995 en el estado de Hidalgo. Se utilizó un diseño de mortalidad proporcional, considerado como una variante del diseño de casos y controles. Los casos fueron aquellas muertes fetales secundarias a defectos del tubo neural y los controles las muertes fetales por otros motivos. Se utilizó ji cuadrada de Pearson para estimar las diferencias entre los casos y controles. Para el riesgo crudo de morir por defectos de cierre del tubo neural se empleó la razón de momios, y para el riesgo ajustado se usó la regresión logística no condicional. Resultados. Se analizaron 3 673 certificados de muerte fetal, identificándose $8.06 \%$ de muertes por defectos del tubo neural; el resto lo constituyeron muertes por otras causas. Se encontró como variables asociadas con la muerte fetal por defectos del tubo neural a los fetos que pesaron menos de 2500 gramos (RM 5.0 , IC 95\% 3.6, 6.7), a los productos del sexo femenino (RM 1.7, IC 95\% 1.3,2.3) y a las muertes ocurridas en el perio do fetal tardío (RM 5.5 IC 95\% 3.8, 8.1). Conclusiones. Los resultados indican que el riesgo de muerte fetal debida a defectos del tubo neural es mayor en productos

\author{
Muñoz-Juárez S,Vargas-Flores H, Hernández-Prado B, \\ López-Ríos O, Ortiz-Espinosa R. \\ Mortality due to neural tube defects \\ and risk factors \\ in Hidalgo, Mexico. \\ Salud Publica Mex 2002;44:422-430. \\ The English version of this paper \\ is available at: http://www.insp.mx/salud/index.html
}

\begin{abstract}
A bstract
Objective.To calculate the risk of fetal death due to neural tube defects and estimate associated factors in the state of Hidalgo, Mexico. Material and Methods. Data were abstracted from death certificates registered during 19901995 in the state of Hidalgo, Mexico. The design was a proportional mortality study, which is considered as a variant of the case control design. Cases were deaths with any type of neural tube defect, and controls were fetal deaths due to other causes. Results. A total of 3673 fetal death certificates were analyzed; $8.06 \%$ had neural tube defects and the remaining died of other causes. Fetal death was associated with fetal weight less than 2500 grams (OR $5.0,95 \% \mathrm{Cl}$ 3.6-6.8), being female $(0 \mathrm{R} 1.7,95 \% \mathrm{Cl} 1.3-2.3)$, and death during the late fetal period $(O \mathrm{R} 5.5,95 \% \mathrm{Cl} 3.8-8.1)$. Conclusions. Results show that the risk of fetal death due to neural tube defects is greater among low birth weight babies, females, and during the late fetal period. The English version of this paper is available at:http://www.insp.mx/salud/ index.html
\end{abstract}

El primer autor agradece a CONACyT el apoyo para la realización de este trabajo.

(1) Coordinación de Investigación, Servicios de Salud de Hidalgo, Universidad Autónoma del Estado de Hidalgo. Pachuca, México.

(2) Dirección General de Regulación de los Servicios de Salud, Secretaría de Salud. México, D.F., México.

(3) Centro de Investigaciones en Salud Poblacional, Instituto N acional de Salud Pública, Cuernavaca, Morelos, México.

(4) Instituto Tecnológico de Estudios Superiores de Monterrey, Campus Ciudad de México. México, D.F., México.

(5) Coordinación de Investigación, Servicios de Salud de Hidalgo, Universidad Autónoma del Estado de Hidalgo. Pachuca, México.

Fecha de recibido: 31 de julio de 2001 • Fecha de aprobado: 10 de mayo de 2002.

Solicitud de sobretiros: Sergio Muñoz-Juárez. A venida México N 0.300 coloniaVilla Aquiles Serdán, 42039 Pachuca, Hidalgo, México. Correo electrónico: smzjz@ hotmail.com 
de bajo peso, en los del sexo femenino y los que ocurren en el periodo fetal tardío. El texto completo en inglés de este artículo está disponible en: http://www.insp.mx/salud/ index.html

Palabras clave: muerte fetal; defectos del tubo neural; malformaciones fetales; estudio de mortalidad proporcional; México
Key words: fo etal mortality; neural tube defects; proportional mortality study design; Mexico
E n el estudio de la muerte, un lugar sustancial lo ocupa la mortalidad perinatal, y dentro de este contexto la muerte fetal, definida como "el producto de la concepción proveniente de un embarazo de 21 semanas o más de gestación, que después de concluir su separación del organismo materno no respira ni manifiesta otros signos de vida como latidos cardiacos o funiculares, o movimientos definidos de músculos voluntarios." $^{11}$

La muerte de los fetos es un fenómeno que en los últimos años ha llamado la atención de la investigación en el ámbito mundial, siendo aún incipiente su estudio en países en desarrollo. ${ }^{2}$ Son varias y diferentes las causas que producen muerte fetal. Dentro de éstas se encuentran los defectos del tubo neural (DTN), definidos como la falta de fusión del tubo neural durante la etapa embrionaria, en la que el tejido nervioso queda expuesto en la superficie. ${ }^{3}$

La etiología de los DTN es multifactorial y éstos son la consecuencia de la interacción de factores ambientales, nutricionales, genéticos, étnicos o de causa desconocida ${ }^{4-8}$ De los estudios hechos, la mayoría son de tipo descriptivo y de muestras de centros hospitalarios. Los DTN representan un riesgo para el producto de la gestación, de enfermedad con secuela o bien la muerte.

La frecuencia de las muertes fetales por DTN, si bien no ha disminuido, se ha mantenido constante en el país y en el estado de Hidalgo; no obstante, se encuentra dentro de las principales causas de muerte fetal. En Venezuela, por ejemplo, los DTN ocupan el tercer lugar en la mortalidad perinatal. ${ }^{4,9}$ De los primeros reportes en la bibliografía que hacen alusión a las malformaciones, y de éstas a los DTN, fue el trabajo realizado por Stevenson y colaboradores, ${ }^{10}$ quienes encontraron a los DTN entre las principales malformaciones observadas. Sánchez y Salazar ${ }^{11}$ en su estudio descriptivo de 58901 nacimientos, reportan una prevalencia de $3 \%$ de malformaciones. Se estudiaron los antecedentes de mortinatos, sexo del producto, peso en intervalos de 500 gramos, madres menores de 20 años de edad y consanguinidad. Las principales malformaciones observadas entre los recién nacidos muertos fueron la anencefalia y la microcefalia.
Wald ${ }^{12}$ hizó un estudio entre el peso del producto y la presencia de espina bífida, y halló una diferencia significativa entre la media de peso de los recién nacidos con DTN frente a los que no tuvieron ningún defecto, observando la existencia de 20 gramos de peso menos entre los niños en relación con las niñas. En Escocia $^{13}$ se estudió la asociación entre DTN y los aspectos sociales y económicos de la madre; se menciona que la frecuencia de anencefalia aumentó durante las décadas de los años 40 y 50. Esto puede ser atribuido a las mujeres que nacieron durante la gran depresión económica de 1926 a 1937. Hacen referencia a la presencia de los factores ambientales y su interacción con los fenómenos de salud y enfermedad de la siguiente generación.

En Beijín -Tianjin, ${ }^{14}$ se estudiaron 210000 nacimientos reportando 1000 casos de DTN, y encontrando marcadas diferencias de DTN entre las áreas rurales y urbanas. Se estimó un riesgo relativo de 2.4 de niños con DTN cuyas madres residían habitualmente en zonas rurales, y que habían emigrado a zonas urbanas. En un estudio de 1243284 nacimientos se encontraron 3404 casos de DTN: 55.6\% fue para la anencefalia; $30.9 \%$, para espina bífida, y $13.6 \%$ para encefalocele. En este estudio se encontraron diferencias significativas entre sexos, siendo las niñas las mayormente afectadas con DTN. La frecuencia de los DTN observados fue mayor en zonas rurales que en urbanas. ${ }^{15}$

Ha sido poca la investigación en México sobre los DTN, y la mayoría de los estudios se han basado en poblaciones hospitalarias. Entre ellas se encuentran trabajos como los de Cobo; ${ }^{16}$ en éste se hizo un análisis descriptivo de 7135 recién nacidos vivos, los cuales $2.08 \%$ presentó alguna malformación. Se observó una mayor proporción de DTN en niñas (2.58\%). Este autor estudió la exposición a radiación en las embarazadas, antecedentes de haber ingerido medicamentos y edad de la madre; además, observó una media de peso para los niños malformados de 3209 gramos, y de 3125 para las niñas. Los DTN reportados fueron el meningocele y la anencefalia, que se encontraron en los lugares $8^{\circ}$ y $10^{\circ}$, respectivamente.

Hernández ${ }^{17}$ estudió a 7791 recién nacidos vivos, encontrando una prevalencia de $12.8 \%$ de defectos 
congénitos. Se analizó el sexo del producto, consanguinidad de la pareja, edad de la madre, paridad y antecedentes de malformaciones. La variable con significancia estadística para la aparición de malformaciones fue la infección viral durante el embarazo. Las malformaciones observadas fueron las del sistema músculoesquelético, urogenital y del sistema nervioso. Canún y Zafra ${ }^{18}$ en el Instituto Nacional de Perinatología, detectaron una prevalencia de $3.3 \%$ de malformados. Los autores describieron las malformaciones por el sexo de los productos, teniendo $3.5 \%$ para los niños y $3.1 \%$ para las niñas. Las malformaciones más frecuentes fueron las del sistema nervioso, labio hendido y pie equinovaro.

Jiménez y colaboradores ${ }^{19}$ revisaron 105825 nacimientos de los cuales $97.1 \%$ nacieron vivos. Se detectaron 2041 casos de malformados, $48.85 \%$ correspondió a las niñas. Se estudió el peso en categorías de 500 gramos, observando como malformaciones más frecuentes a la luxación congénita de cadera, del corazón y defectos del cierre del tubo neural. Es interesante mencionar la mayor frecuencia de DTN encontrada en las niñas, y de éstas, en las que pesaban menos de 2500.

De algunas conclusiones de Registro y Vigilancia Epidemiológica de Malformaciones Congénitas ${ }^{5}$ se menciona que en México uno de cada 50 recién nacidos vivos, y uno de cada nueve recién nacidos muertos presentan alguna malformación, que puede ser de tipo letal como la anencefalia. En Monterrey ${ }^{20}$ se estudió a 9675 recién nacidos vivos con malformaciones, la mayor incidencia de éstas correspondieron a las del sistema nervioso central (encefalocele, espina bífida con hidrocefalia e hidrocefalia), seguidas de las de tipo cardiovascular y del sistema músculo-esquelético; se menciona la importancia de realizar estudios en recién nacidos muertos.

De las investigaciones hechas en México respecto de los DTN, se puede determinar que es escasa la investigación realizada en este tema, comparada con la de otros países, y que dicha investigación es en su mayoría de tipo descriptiva. De acuerdo con los antecedentes descritos, de los DTN se ha estudiado, por un lado, su frecuencia, y a algunos otros se les ha relacionado con diferentes tipos de exposición como los extremos de la edad reproductiva de las madres, el peso de los productos, si estos se encuentran por arriba o abajo de los 2500 gramos, la edad gestacional, el nivel socioeconómico de las madres, la raza, la variación de las anomalías entre el sexo de los productos, las zonas rurales y urbanas, y la consanguinidad de los padres. Y por otro lado, los antecedentes de tabaquismo, alcoholismo y ocupación de los padres. De la misma manera los datos correspondientes a la identificación de carencia de algunos micronutrimentos como el ácido fólico y la vitamina A, etcétera.

De las investigaciones en México surgen dos contextos por determinar; el primero, en relación con la poca investigación en este tema, comparado con la de otros países, y el segundo, que la investigación realizada es en su mayoría de tipo descriptivo. Por este motivo surge esta nueva propuesta en el amplio campo de los DTN, de cuantificar el riesgo de muerte fetal por esta causa. Los objetivos de esta investigación fueron estimar el riesgo de muerte fetal secundaria a defectos del cierre del tubo neural por medio de la información registrada en los certificados de muerte fetal durante los años de 1990 a 1995 en el estado de Hidalgo, e identificar las variables biosociodemográficas secundarias de DTN, y asociadas con el riesgo de muerte fetal.

\section{Material y métodos}

El presente estudio se realizó en el estado de Hidalgo, México, en el año 1999-2000 con la información proveniente de los certificados de defunción fetal (CDF) de un periodo de seis años (1990-1995). Se utilizó un diseño de mortalidad proporcional, que involucra sólo a muertos, y es utilizado cuando se tienen datos acerca de la mortalidad de interés. Las muertes son identificadas en una población definida donde existe el riesgo de desarrollar el factor de interés, a este diseño se le considera como un tipo especial de casos y controles, ya que se tiene información del factor de estudio de todas las muertes en una población. Los estudios proporcionales de mortalidad incluyen únicamente a sujetos muertos, la proporción de sujetos muertos que estuvieron expuestos, es comparada con una correspondiente proporción de sujetos que no lo estuvieron. ${ }^{21-23}$

Se incluyeron a los CDF del estado durante el periodo 1990-1995, correspondiente a los 84 municipios que conforman la entidad. Los fetos muertos con defunción secundaria a DTN (anencefalia, craneorraquisquisis y espina bífida con hidrocefalia) fueron designados como casos, los controles fueron los fetos que murieron por otra causa..$^{21-23}$

Para la clasificación de los DTN se utilizó la Clasificación Internacional de Enfermedades en su novena revisión, ${ }^{24}$ (la razón de utilizarla fue porque los CFD estuvieron clasificados con esta revisión). En contraposición se clasificó como muerte fetal debida a otras causas a las defunciones que el certificado de muerte fetal no señalara como causa básica de los DTN. Se eliminaron los certificados de defunción que presentaron datos incompletos, o cuya residencia habitual de 
la madre no fuera en el estado de Hidalgo; bajo estos criterios se eliminó $16.8 \%$ de los certificados que correspondieron a los casos, y $19.2 \%$ de los controles.

Se determinó como variables de exposición al peso menor de 2500 gramos, el sexo femenino, al nacimiento durante el periodo fetal tardío, y a las madres menores de 19 años con residencia habitual en municipios de alta marginación. Estas variables fueron elegidas por ser algunas de las estudiadas por otros autores; ${ }^{17-20,25-36}$ además, porque pueden ser obtenidas del $\mathrm{CDF}$, con el fin de observar su comportamiento y variabilidad, en el estado. La población de casos y controles, expuestos y no expuestos en las categorías de interés fue extraída de la población base de certificados de muerte fetal. ${ }^{37-38}$

En cuanto al índice de marginación, se utilizó el diseñado por el Consejo Nacional de Población (Conapo). ${ }^{39}$ Este índice mide el grado de marginación municipal de cada entidad federativa, analizando la marginación social a través de sus dimensiones, formas específicas e intensidades, y finalizando con una valoración unitaria del conjunto de carencias.

La aplicación del concepto de marginación municipal, según este índice, contempla cuatro dimensiones: vivienda, ingresos monetarios, y educación y distribución de la población. Conapo evalúa dichas dimensiones por medio de la ausencia de agua entubada, energía eléctrica y drenaje, viviendas con piso de tierra y de tamaño inadecuado a las necesidades del hogar. Respecto de los ingresos monetarios, explora la población que percibe hasta dos salarios mínimos. En la dimensión de educación los parámetros son población analfabeta y con primaria incompleta. Finalmente, se explora la población en localidades de menos de 5000 habitantes.

Según el resultado de este índice, los ayuntamientos se clasifican en cinco diferentes categorías: 1) municipios de muy baja marginación, 2) baja, 3) media, 4) alta y 5) muy alta marginación. De acuerdo con este índice, las mujeres fueron clasificadas según su residencia habitual, en el municipio correspondiente según el grado de marginación municipal. Mediante el índice de marginación del Conapo, no sólo se clasifica a los municipios de la entidad, sino que las entidades federativas se incluyen en el contexto nacional para su clasificación de marginación.

Se estudió el comportamiento de las variables biosociodemográficas, realizando un análisis univariado. En éste se calculó la prevalencia de exposición de las variables de interés. Se estimó la razón de momios cruda, para evaluar la asociación entre los factores de estudio y la muerte fetal por DTN con intervalos de confianza a 95\% de acuerdo con el diseño utilizado. ${ }^{21-23}$ Para el análisis multivariado se ajustaron mo- delos de regresión logística no condicional ${ }^{21,22}$ con el fin de estimar la razón de momios ajustada por las variables potencialmente confusoras. ${ }^{37}$

El criterio para identificar a una variable como confusora consistió en comparar el estimado del efecto obtenido de los datos crudos con el correspondiente de los datos estratificados, y obteniendo un estimado global del efecto utilizando la ponderación de Mantel - Haenzel.

En la selección de las variables se utilizaron aquellas que mejor explicaran el fenómeno de los DTN. No se hizo ninguna transformación de las variables. Se estimó el riesgo de que madres muy jóvenes (hasta 19 años de edad) tuvieran un hijo que muriera por un DTN, con relación a las madres de 20 años y más; asimismo, se estimó el riesgo de aquellas madres que vivieron en municipios de muy alta marginación, respecto de los otros municipios con diferentes grados de marginación (de alta hasta muy baja).

De igual forma se evaluó la interacción entre la edad menor de 20 años y la residencia en municipios de muy alta marginación, con su correspondiente contraparte. Los municipios se clasificaron en los cinco grados de marginación, dependiendo la residencia habitual de la madre, según constatara en el certificado de defunción.

\section{Resultados}

Se analizaron 3673 muertes fetales de las cuales $8.06 \%$ fueron secundarias a DTN (cuadro I). Con respecto a los 84 municipios que conforman la entidad, se clasificaron según su grado de marginación utilizando el índice ya mencionado, esta distribución se muestra en el cuadro II. Utilizando la ji cuadrada de Pearson, se ana-

\section{Cuadro I \\ Proporción de muertes fetales por defectos del Tubo Neural en el estado de Hidalgo. MÉxıCO, 1990-1995}

\begin{tabular}{lccc} 
Año & $\begin{array}{c}\text { Número de } \\
\text { muertes fetales }\end{array}$ & \multicolumn{2}{c}{ Muertes fetales por defectos del tubo neural } \\
\cline { 3 - 4 } & & Número & Proporción \\
1990 & 605 & 66 & $10.91 \%$ \\
\hline 1991 & 560 & 32 & $5.71 \%$ \\
\hline 1992 & 643 & 37 & $5.75 \%$ \\
\hline 1993 & 631 & 52 & $8.24 \%$ \\
\hline 1994 & 641 & 59 & $9.20 \%$ \\
\hline 1995 & 593 & 50 & $8.43 \%$ \\
\hline Total & 3673 & 296 & $8.06 \%$
\end{tabular}

Fuente: Certificados de muerte fetal, Hidalgo, México, 1990-1995 


\section{Cuadro II \\ DISTRIBUCIÓN MUNICIPAL POR GRADO DE MARGINACIÓN en el estado de Hidalgo, México, 1990-1995}

\begin{tabular}{lcc} 
Grado de marginación & Municipios & Porcentaje \\
Muy alta & 12 & 14.29 \\
\hline Alta & 31 & 36.90 \\
\hline Media & 15 & 17.86 \\
\hline Baja & 23 & 27.38 \\
\hline Muy baja & 3 & 3.57 \\
\hline Total & 84 & 100.00
\end{tabular}

Fuente: Indice de marginación municipal. Consejo N acional de Población

lizaron las diferencias entre las variables que conforman los CDF y los DTN. Estos resultados se pueden apreciar en el cuadro III.

Del total de muertes fetales, se encontró que $56.47 \%$ de los fetos pesaron menos de 2500 gramos y que de éstos $11.57 \%$ había muerto por un DTN en esta categoría de peso, encontrando un riesgo de morir por DTN en quienes tenían menos de 2500 gramos ( $R M$ 5.0, IC 95\% 3.6-6.7).

En lo referente al sexo de los fetos, del total $44.84 \%$ correspondió al sexo femenino, de éstas $10.87 \%$ tuvo como diagnóstico un DTN, con una razón de 1.5 mujeres con DTN por cada varón, por lo que en los casos la mayor proporción se observó en las niñas $(60.47 \%)$ encontrando en ellos un riesgo de morir por DTN (RM 1.7, IC 95\% 1.3-2.3). Por otra parte, el total de muertes fetales fue clasificado en los periodos fetal temprano y tardío, correspondiendo $70.65 \%$ a este último periodo, de éstos $10.17 \%$ murió a consecuencia de DTN y se observó un riesgo de morir para los del periodo fetal tardío (RM 5.5, IC 95\% 3.8-8.1).

El $19.74 \%$ de los fetos muertos correspondió a madres menores de 20 años de edad con residencia en municipios de muy alta marginación, de éstos $9.24 \%$ murió por algún DTN. No se encontró asociación entre la mortalidad debida a DTN en aquellos fetos cuyas madres tenían menos de 20 años y que vivieron en municipios de muy alta marginación (RM 1.2, IC $95 \%$ 0.90, 1.6). La principal causa de los DTN fue la anencefalia, con $86.8 \%$; en segundo lugar la espina bífida con hidrocefalia, con $6.4 \%$; en tercero la craneorraquisquisis, con $4.7 \%$, y en cuarto lugar la espina bífida, que aportó $2.0 \%$. La razón de momios cruda al presentar DTN para los productos con peso menor de 2500 gramos es 3.6 veces mayor con relación a los productos con peso superior a 2500 gramos incrementándose ésta al ajustar por variables sexo del producto, madres menores de 20 años de edad con residencia en municipios con muy alta marginación y que la muerte corresponda a aquéllas ocurridas en el periodo fetal tardío alcanzando hasta cinco veces más el riesgo de presentar un DTN con relación a aquellos productos con peso superior a 2500 gramos (cuadro IV).

\section{Discusión}

Los DTN tienen en el ámbito nacional un comportamiento que se considera de alta frecuencia en el periodo analizado, ya que se reportan en el quinto lugar de muerte fetal, excepto en 1994, cuando ocupó el cuarto lugar. De la misma manera se puede considerar como de alta frecuencia la mortalidad por DTN en el estado, puesto que esta causa siempre se encontró entre las primeras 10 de muerte fetal, ocupando siempre las principales causas de mortalidad, la correspondiente a la del feto afectado por complicaciones de la placenta, del cordón umbilical y de las membranas (clave CIE 761) seguido por otras afecciones y las mal definidas que se originan en el periodo perinatal (clave CIE 779). Los DTN se encontraron entre los lugares cuarto y quinto de este periodo.

El presente trabajo pretendió identificar la magnitud, trascendencia y vulnerabilidad del evento, con el propósito de explorar y determinar los factores que puedan explicar la muerte fetal por esta causa. De las variables analizadas el aspecto que sobresale es el mayor riesgo de morir de las niñas, compatible con los resultados en India, ${ }^{26}$ China, ${ }_{1}^{14}$ Nigeriaa $^{28}$ y Palestina. ${ }^{34}$ Este hallazgo es tambien compatible con el estudio mexicano de Jiménez, ${ }^{19}$ en el cual se encontró mayor frecuencia de DTN en el sexo femenino. El presente estudio encontró una razón de 1.5 mujeres con DTN similar a la razón reportada por Dudin en Palestina, sin embargo existen otros estudios que registran un mayor predomino de esta malformación en el sexo masculino.

Este trabajo no analiza lo que ocurre desde la primera hasta la vigésima semana de gestación, existiendo la posibilidad de una mayor mortalidad de varones con DTN en las primeras veinte semanas de vida. Estos hallazgos causan controversia, y proporcionan la pauta para continuar la investigación, que incluya a los productos de la concepción menores de 20 semanas de gestación.

Con respecto a la edad materna, aun cuando otros estudios han mostrado riesgo de morir de los fetos cuando las madres tienen menos de 20 años, como lo demuestran los estudios realizados en Beijín, ${ }^{14}$ India ${ }^{26}$ y Estados Unidos de América, ${ }^{25,33}$ en estos resultados no se encontraron dichos riesgos. No obs- 


\begin{tabular}{|c|c|c|c|c|c|c|c|c|c|}
\hline $\begin{array}{l}\text { DeSCRI } \\
\text { DE MUERTE FETAL POR C }\end{array}$ & $\begin{array}{l}\text { RIPCIÓN DE L } \\
\text { CASOS DE D }\end{array}$ & $\begin{array}{l}\text { A POBL } \\
\text { EECTO }\end{array}$ & CIÓN & $\begin{aligned} & \mathbf{C u} \\
& \text { ESTUDIA } \\
& \text { UBO } \mathbf{N}\end{aligned}$ & $\begin{array}{l}\text { III } \\
\text { JARIABLES INCLUIDAS EN } \\
\text { L Y EL GRUPO CONTROL. }\end{array}$ & $\begin{array}{l}\text { EL CERTIFICA } \\
\text { HidAlgo, M }\end{array}$ & $\begin{array}{l}\text { DO } \\
\text { ÉXICO, }\end{array}$ & 1990 & 1995 \\
\hline Variable & $\begin{array}{l}\text { asos de defectos } \\
\text { del tubo neural }\end{array}$ & Control & Total & Valor P & Variable & $\begin{array}{l}\text { asos de defectos } \\
\text { del tubo neural }\end{array}$ & Control & Total & Valor $\mathrm{P}$ \\
\hline Sexo & & & & & Q uién atendió el parto & & & & \\
\hline Masculino & 39.53 & 56.53 & 55.16 & 0.000 & Médico & 90.54 & 91.06 & 91.02 & 0.234 \\
\hline Femenino & 60.47 & 43.47 & 44.84 & & Enfermera & 2.03 & 1.01 & 1.09 & \\
\hline & & & & & Partera & 3.72 & 2.87 & 2.94 & \\
\hline Peso & & & & & 0 tro & 3.72 & 5.06 & 4.96 & \\
\hline$<2500$ & 81.08 & 54.31 & 56.47 & 0.000 & & & & & \\
\hline$>2500$ & 18.92 & 45.69 & 43.53 & & $\begin{array}{l}\text { El parto fue } \\
\text { N ormal }\end{array}$ & 48.99 & 58.19 & 57.45 & 0.002 \\
\hline Periodo fetal & & & & & Complicado & 51.01 & 41.81 & 42.55 & \\
\hline Tardío & 89.19 & 69.03 & 70.65 & 0.000 & Derechohabiencia & & & & \\
\hline Temprano & 10.81 & 30.97 & 29.35 & & $\mathrm{~N}$ inguna & 73.99 & 71.25 & 71.47 & \\
\hline & & & & & IMSS & 17.23 & 18.03 & 17.97 & \\
\hline Embarazo & & & & & ISSSTE & 3.72 & 4.47 & 4.41 & \\
\hline Unico & 97.64 & 95.50 & 95.67 & 0.195 & Pemex & 0.0 & 0.38 & 0.35 & 0.442 \\
\hline Gemelar & 2.03 & 4.18 & 4.0 & & Fuerza armada & 0.0 & 0.18 & 0.16 & \\
\hline Más de tres & 0.33 & 0.32 & 0.33 & & O tro & $\begin{array}{l}1.35 \\
3.72\end{array}$ & $\begin{array}{l}0.59 \\
5.09\end{array}$ & $\begin{array}{l}0.65 \\
4.98\end{array}$ & \\
\hline Recibió atención prenatal & & & & & & & & & \\
\hline Sí & 59.80 & 62.10 & 61.91 & 0.435 & Estado civil de la madre & & & & \\
\hline No & 40.20 & 37.90 & 38.09 & & Soltera & 5.74 & 8.26 & 8.06 & \\
\hline & & & & & Casada & 57.09 & 56.35 & 56.41 & \\
\hline Evolución del embarazo & & & & & Unión libre & 36.82 & 34.11 & 34.33 & 0.438 \\
\hline Normal & 62.84 & 62.81 & 62.81 & 0.992 & Separada & 0.34 & 1.01 & 0.95 & \\
\hline Complicado & 37.16 & 37.19 & 37.19 & & Divorciada & 0.0 & 0.09 & 0.08 & \\
\hline & & & & & Viuda & 0.0 & 0.18 & 0.16 & \\
\hline Momento en que ocurrió la mu & nuerte & & & & & & & & \\
\hline Antes del parto & 77.03 & 83.86 & 83.31 & 0.002 & Escolaridad & & & & \\
\hline Después del parto & 22.97 & 16.14 & 16.69 & & Ninguna & 11.49 & 14.33 & 14.10 & \\
\hline & & & & & 3 a 5 años & $\begin{array}{l}18.24 \\
14.19\end{array}$ & $\begin{array}{l}13.59 \\
13.83\end{array}$ & $\begin{array}{l}13.91 \\
13.86\end{array}$ & \\
\hline $\begin{array}{l}\text { Sitio en que ocurrió la muerte } \\
\text { Unidad médica }\end{array}$ & & & & & Primaria completa & 31.08 & 24.19 & 24.75 & 0.003 \\
\hline $\begin{array}{l}\text { Unidad médica } \\
\text { Hogar }\end{array}$ & 91.21 & 89.81 & 89.93 & 0.722 & Secundaria o equivalente & 15.88 & 18.63 & 18.40 & \\
\hline $\begin{array}{l}\text { Hogar } \\
0 \text { tro }\end{array}$ & 6.76 & 8.05 & 7.95 & & Preparatoria o equivalente & 6.76 & 8.82 & 8.66 & \\
\hline 0 tro & 2.03 & 2.13 & 2.12 & & Profesional & 1.35 & 4.29 & 4.06 & \\
\hline & & & & & Se ignora & 1.01 & 2.31 & 2.21 & \\
\hline
\end{tabular}

Fuente: Certificados de muerte fetal, Hidalgo. México, 1990-1995

IMSS: Instituto Mexicano del Seguro Social

ISSSTE: Instituto de Seguridad y Servicios Sociales de los Trabajadores del Estado Pemex: Petróleos Mexicanos

tante otros autores refieren que los extremos de la vida reproductiva pueden considerarse como de riesgo para la parición de DTN. Al respecto los estudios que mencionan en sus resultados la edad muy joven de la madre son los de Lian en China, ${ }^{14}$ los de Kulkarni en India, ${ }^{26}$ Haddow $^{31}$ y Canfield en Houston. ${ }^{33}$

Hecho interesante son los hallazgos reportados por Haddow $^{31}$ en 1993, entre los cuales se menciona un riesgo hasta 7.3 veces mayor de muerte por DTN cuando las madres son menores de 20 años, en comparación con otros grupos de edad, y el de Canfield en Texas. ${ }^{33}$ Entre las posibles explicaciones se encuentra la biológica, sustentanda en el hecho de que estas madres no han alcanzado su madurez biológica ${ }^{40}$ para la procreación sin malformaciones; sin embargo, es necesario destacar que el evento es multifactorial, y que únicamente la edad no podría explicar la presencia de los DTN por sí mismos.

Algo parecido ocurrió con el nivel de marginación de las madres, el muy bajo presentó riesgo en el aná- 


\section{Cuadro IV \\ Proporción de muertes fetales por defectos del Tubo Neural, razón de momios Cruda Y AJUSTADA DE ACUERDO CON LAS VARIABLES del estudio, Hidalgo, México, 1990-1995}

\begin{tabular}{|c|c|c|c|c|c|}
\hline $\begin{array}{r}N \\
\text { mue } \\
p\end{array}$ & $\begin{array}{l}\text { úmero de } \\
\text { rtes fetales } \\
\text { or DTN* }\end{array}$ & $\begin{array}{l}\text { Razón de } \\
\text { momios } \\
\text { cruda }\end{array}$ & IC $95 \%$ & $\begin{array}{c}\text { Razón de } \\
\text { momios } \\
\text { ajustada* }\end{array}$ & IC 95\% \\
\hline Peso al nacer & & & & & \\
\hline$<2500 \mathrm{gr}$. & 240 & 3.6 & $2.6-4.8$ & 5.0 & $3.6-6.7$ \\
\hline$>2500 \mathrm{gr}$ & 56 & 1.0 & & & \\
\hline Sexo & & & & & \\
\hline Femenino & 179 & 1.9 & $1.5-2.5$ & 1.7 & $1.3-2.3$ \\
\hline Masculino & 117 & 1.0 & & & \\
\hline Periodo fetal & & & & & \\
\hline Tardío & 264 & 3.7 & $2.5-5.3$ & 5.5 & $3.8-8.1$ \\
\hline Temprano & 32 & 1.0 & & & \\
\hline Muy alta marginación & 22 & 1.6 & $1.01-2.5$ & 1.22 & $0.39-3.7$ \\
\hline $\begin{array}{l}\text { Alta a muy baja } \\
\text { marginación }\end{array}$ & 274 & 1.0 & & & \\
\hline Madre de 19 años de e & & & & & \\
\hline 0 menos & 49 & 1.06 & $0.70-1.46$ & 0.82 & $0.24-2.7$ \\
\hline $\begin{array}{l}\text { Madre de } 20 \text { años de e } \\
\text { y más }\end{array}$ & $\begin{array}{l}\mathrm{dad} \\
247\end{array}$ & 1.0 & & & \\
\hline $\begin{array}{l}\text { Madres menores de } 20 \\
\text { años de edad que viven } \\
\text { en municipios de muy } \\
\text { alta marginación }\end{array}$ & 67 & 1.2 & $0.90-1.6$ & 1.08 & $0.80-1.4$ \\
\hline $\begin{array}{l}\text { Madres mayores de } 19 \\
\text { años de edad que no } \\
\text { viven en municipios de } \\
\text { muy alta marginación }\end{array}$ & 229 & 1.0 & & & \\
\hline * DTN : defectos del tu & oo neural & & & & \\
\hline te: Certificados & ertef & Hidal & óxi & 90 & \\
\hline
\end{tabular}

lisis crudo, el cual se pierde en el análisis multivariado, a diferencia de lo encontrado en otros estudios como el de Jiménez. ${ }^{19}$ Respecto del nivel socioeconómico es necesario mencionar que aun cuando los CDF no permiten esta estimación, el índice de marginación permite evaluarlo de manera indirecta.

Aunque estadísticamente se observa una diferencia escasamente marginal, de la muy alta marginación, esta diferencia se pierde al hacer la interacción con las mujeres jóvenes (cuadro IV). Al parecer el índice de marginación podría ser un indicador indirecto del nivel socioeconómico cuando no se cuenta con datos más precisos para medir éste; de esta manera surge esta propuesta para que dicho índice sea utilizado en otros estudios y se constate su utilidad o inconveniencia en estudios observacionales.
Es necesario señalar que el no haber encontrado significancia estadística en las variables de muy alta marginación, en las madres menores de 20 años de edad y en la interacción analizada, tal vez ocurra no por la falta de asociación entre la causa y la presencia de DTN, sino más bien por la falta de poder estadístico, situación que puede presentarse cuando se hacen análisis de bases secundarias de datos.

Sin embargo, con los datos de las variables analizadas, aun cuando no se demostró asociación entre estas causas y los DTN, se pueden explicar de manera indirecta, de acuerdo con lo señalado por Smith, ${ }^{29}$ los riesgos que corren los productos de madres desnutridas están asociados con el nivel socioeconómico y el grado de marginación, ya que la probabilidad de encontrar mayor número de madres con carencias nutricionales podría ser más frecuente en estas zonas de la entidad, y la probabilidad de desarrollar anomalías estructurales es mayor que en las madres bien nutridas.

La residencia habitual en municipios de muy alta marginación con menores posibilidades de desarrollo socioeconómico podrían estar incidiendo en menor accesibilidad y disponibilidad de nutrientes; esto de alguna manera podría contribuir a la aparición de DTN. Sería difícil precisar qué ocurre detrás de la pobreza que incrementa la posibilidad de que aparezcan los DTN, siendo tal vez alguna carencia nutricional, ${ }^{41}$ el aislamiento geográfico, o la exposición a algún agente químico ${ }^{42}$ lo que propicie este efecto. Todas éstas, constituyen situaciones que en un futuro se podrían abordar con diferentes líneas de investigación. No obstante, los resultados sólo pueden ofrecer especulaciones, según lo encontrado en las investigaciones previas de DTN, por lo que sería importante la realización de evaluaciones más sensibles y específicas del nivel socioeconómico o del estado nutricio de las madres, así como el estudio de poblaciones con el tamaño muestral suficiente para disminuir la posibilidad de cometer un error tipo II, por falta de poder estadístico.

Con respecto a la mayor probabilidad de morir por DTN si el feto pesa entre 500 y 2500 gramos los resultados concuerdan con otros estudios; ${ }^{4,11,15,26,28,30}$ es importante mencionar los resultados de Kulkarni ${ }^{26}$ y Airede $^{28}$ quienes encontraron diferencias en los DTN entre las categorías de mayor y menor de 2500 gramos del peso de los fetos.

Los productos pueden tener un bajo peso debido a las propias malformaciones, ya que si a un producto con anencefalia le falta parte de la bóveda craneal y de tejido cerebral, se produce bajo peso. Sin embargo ésta no da respuesta a otros DTN como los defectos de cierre de la columna. Por ello, es necesario investigar más a 
fondo esta variable para observar su comportamiento en la frecuencia de los DTN.

Respecto del mayor riesgo que tiene el feto de morir por DTN en el periodo fetal tardío, son pocos los estudios en la literatura al respecto, ya que sólo se hace mención de la edad gestacional como un hallazgo, sin que se dé una explicación de su relación con los DTN. ${ }^{10,17,27,34,43,44}$

Por otro lado, algunas variables mostradas en el cuadro III que presentaron diferencias estadísticas entre los grupos con DTN y el grupo de comparación, no fueron consideradas dentro del análisis para estimar el riesgo de morir por un DTN, ya que estas no se encuentran, por lo menos a la luz de los conocimientos actuales, como causa de DTN.

De los resultados observados, y considerando las limitaciones de la población estudiada (por la fuente de información), podemos asumir algunas conclusiones: que los DTN se presentan con mayor frecuencia en las niñas durante la etapa fetal, lo que estaría condicionando una mayor mortalidad en este sexo, sin embargo, es necesario seguir investigando qué pasa con los productos de menos de 20 semanas de gestación, con el fin de confrontar estos resultados.

En lo referente al periodo fetal pudiera pasar algo parecido, ya que tal vez los fetos con DTN podrían tener una mayor mortalidad de manera inicial en el periodo fetal temprano, y que, aunado al subregistro de la muerte en esta etapa, condicionaría una aparente mayor mortalidad en la etapa fetal tardía, o pudiera ocurrir que las DTN no provoquen la muerte de los fetos de manera temprana, sino hasta que estos llegan a la etapa fetal tardía provocando una mayor mortalidad en este periodo. Debido a la naturaleza con que se desarrolló esta investigación podemos sugerir algunas recomendaciones, como las de emprender de manera paulatina pruebas de tamizaje para un diagnóstico oportuno de malformaciones fetales por medio de ultrasonido, como método no invasivo ${ }^{45,46}$ e inofensivo para el producto.

Se recomienda la creación de consejos genéticos para aquellas parejas que tienen antecedentes familiares de malformaciones o con hijos malformados previos. Incrementar dichas acciones en parejas consanguíneas con el fin de evaluar tempranamente dicho riesgo.

Mejorar la accesibilidad, disponibilidad y uso de metodología anticonceptiva para la prevención de embarazos con alto riesgo de que sufran alguna malformación. De manera preventiva, suplementar en forma temprana a las mujeres embarazadas con complejos vitamínicos y ácido fólico. ${ }^{47-50}$ Finalmente, un incremento en la inversión para la realización de in- vestigaciones con mejores metodologías sobre las malformaciones congénitas.

\section{Referencias}

1. Secretaría de Salud. N orma 0 ficial Mexicana N O M-007-SSA2-1993, para la atención de la mujer durante el embarazo, parto, puerperio y del recién nacido. Criterios y procedimientos para la prestación de servicio. Diario 0 ficial de la Federación, 6 de enero de 1995.

2. Langer A. Enfoques de la investigación perinatal. Salud Publica Mex 1988;30(1):43-46.

3. Moore KL. Embriología clínica. 6 a edición. México, D.F.: McG raw Hill, 1999:304-341.

4. Cedeño RR, León A, Romero R. Epidemiología de las malformaciones congénitas externas en una maternidad en Venezuela. Bol Med Hosp Infant Mex 1996;53:117-122.

5. Mutchinick O, Lisker R, Babinski V. Programa mexicano de registro y vigilancia epidemiológica de malformaciones congénitas. Salud Publica Mex 1988;30:88-100.

6. Hernández JL, Cortés G G, AldanaVC, Ramírez HA. Incidencia de malformaciones congénitas externas en el hospital de ginecopediatría no. 48 en León Guanajuato. Bol Med Hosp Infant Mex 1991;48:717-721.

7. Cortés CR, Marín RR,A guilar N S. Epidemiología de las malformaciones congénitas externas. G inecol 0 bstet Mex 1986;54:311-321.

8. Holmes LB. Congenital malformations. N Engl J Med 1980;295: 204207.

9. Castilla EE, Mutchinick O, Paz J, Muñoz E, Gelman Z. Estudio latinoamericano sobre malformaciones congénitas. Bol 0 ficina Sanit Panam 1974:76(6):494-501.

10. Stevenson AC, Johnston HA, Stewart MIP. Congenital malformations:A report of series of consecutive births en 24 centers. Bull W orld Health O rgan 1966;34(Suppl):9-127.

11. Sánchez 0 , Salazar A, Ramírez N , Alvarez M. Epidemiología de malformaciones congénitas en el hospital Ruiz Páez de Ciudad Bolívar: una experiencia de 10 años. Invest clin 1989;30(3):159-172.

12.W ald $\mathrm{N}$ J, Cukle HS, Boreham J;Althouse R. Birth weight of infant with spina bifida cystica. Br J 0 bstet Gynaecol 1980;87(7):578-581.

13. Baird D. Environment and reproduction. Br J O bstet Gynaecol 1980;87(12):1057-1067.

14. Lian ZH, Yang HY, Li Z. N eural tube defects in Beijin-Tianjin area of China. Urban-rural distribution and some other epidemiological characteristics.J Epidemiol Community Health 1987;41(3):259-262.

15. Xiao KZ. Epidemiology of neural tube defects in China. Chung Hua I Hsueh Tsa Chih 1989;69(4):189-191.

16. C obo A, GarcíaVA, Barriga A, López S, N ájera J. Frecuencia de malformaciones congénitas en recién nacidos vivos en León Guanajuato. Rev Invest Clin 1978;30:277-281.

17. Hernández A, Corona R, Martínez B,A guirre N , Cantú J. Factores prenatales y defectos congénitos en una población de 7791 recién nacidos consecutivos. Bol Med Hosp Infant Mex 1983:40(7):363-366.

18. C anún SS, Z afra RG. D etección de malformaciones congénitas externas. Bol Med Hosp Infant Mex 1984;41(1):21-24.

19. Jiménez BE, Salamanca GF, MartínezAS, Bracho SM. Estudio de malformaciones congénitas en 105825 nacimientos consecutivos. Bol Med Hosp Infant Mex 1985;42:744-748.

20. Arredondo AG, Rodríguez BR, Treviño AG, Arreola $A B$, Astudillo CG, Russildi JM. Malformaciones congénitas en recién nacidos vivos. Bol Med Hosp Infant Mex 1990;47(12):882-827.

21. Rothman KL. Modern epidemiology. Boston: Little Brown, 1986:51-76. 
22. Kleinbaum DG, Kupper LL, Morgenstern H. Epidemiologic research. Principles and quantitative methods. N uevaYork (N Y):Van N ostrand Runhold, 1982:81-83.

23. Miettinen $\mathrm{OS}, \mathrm{W}$ ang J.An alternative to the proportionate mortality ratio.Am J Epidemiol 1981;114(1):144-148.

24. O rganización Mundial de la Salud. Clasificación Internacional de enfermedades. 9a revisión. Ginebra: O MS, 1975:442-465.

25. Sever LE.An epidemiologic study of neural tube defects in LosAngeles County II. Etiologic factors in an area with low prevalence at birth. Teratology 1982;25(3):323-334.

26. Kulkarni ML, Mathew MA, ReddyV.The range of neural tube defects in Southern India. Arch D is Child 1989;64(2):201-204.

27. Crandall BF, Robinson L, Grau P. Risks associated with an elevated maternal serum alpha-fetoprotein level. Am J O bstet Gynecol 1991;165(3):581-586

28. Airede KI. Neural tube defects in the middle belt of Nigeria. J Trop Pediatr 1992;38(1):27-30.

29. Smith N C. Detection of the fetus at risk. Eur J Clin Nutr 1992;46 (Suppl 1):S1-S5.

30. 0 gataAJ, Camano L, Brunoni D. Perinatal factors associated with neural tube defects (anencephaly [correction of anencephaly], spina bifida and encephalocele). Rev Paul Med 1992;110(4):147-151.

31. Haddow JE, Palomaki GE, Holman MS.Young maternal age and smoking during pregnancy as risk factor for gastroschisis. Teratology 1993;47(3): 225-228.

32. Kallen B, Cocchi G, Knudsen LB, Castilla EE, Robert E, Daltveit AK. International study of sex ratio and twinning of neural tube defects. Teratology 1994;50(5):322-331.

33. C anfield MA,A nnegers JF, Brender JD, C ooper SP, G reenberg F. H ispanic origin and neural tube defects in Houston/Harris County, Texas. II. Risk factors. Am J Epidemiol 1996;143(1):12-24.

34. Dudin A. N eural tube defect among Palestinians:A hospital-based study. Ann Trop Paediatr 1997; 17(3):217-222.

35. W ang $Y, Z$ hu J, W u Y. Dynamic variation of incidence of neural tube defects during 1988 to 1992 in China. Chung Hua I Hsueh Tsa Chih 1998:32(6):369-371.

36.W asserman CR, Shaw G M, Selvin S, G ould JB, Syme SL. Socioeconomic status, neighborhood social conditions, and neural tube defects. Am J Public Health 1998;88(11):1674-1680.
37. Schlesselman JJ. Case control studies. N uevaYork (N Y): 0 xford University Press, 1982: 227-280.

38.- Miettinen $0 \mathrm{~S}$. Theorical epidemiology. Principles of occurrence research in medicine. N ueva York (N Y): John W iloy \& Sons, 1985:260.

39. Consejo $\mathrm{N}$ acional de Población. Indice de marginación municipal. México, D.F.: Conapo, 1995:590-596.

40. $O$ lausson PO, C nattingius $\mathrm{S}$, Haglund B. Teenage pregnancies and risk of late fetal death and infant mortality. $\mathrm{Br} J \mathrm{O}$ bstet Gynaecol 1999; 106(2):116-121.

41. Rodríguez MM, Guerrero JF, Parra Q M, Segura MJ, Levario CM, Sotelo El. La deficiencia de ácido fólico y su asociación con defectos del tubo neural en el norte de México. Salud Publica Mex 1998;40(6):474-480.

42. Croen LA, Shaw GM, Sanbonmatsu L, Selvin S, Buffler PA. Maternal residential proximity to hazardous waste sites and risk for selected congenital malformations. Epidemiology 1997;8(4):347-354.

43. 0 rganización Mundial de la Salud. Factores genéticos y malformaciones congénitas. Informe de un grupo de científicos de la O MS 1970. Ginebra: OMS, 1970; informe técnico no.438.

44. Borman B, Cryer C. Fallacies of international and national comparisons of disease occurrence in the epidemiology of neural tube defects. Teratology 1990;42(4):405-412.

45. Bernaschek G, Stuempflen Y, D eutinger J. The value of sonographic diagnosis of fetal malformations: $D$ ifferent results between indication based and screening based investigations. Prenat Diagn 1994;14(9):807-812.

46. Hori E, Koyanagi T,Yoshizato T, Maeda H, Satoh S, Suita S. How antenatal ultrasound diagnosis of congenital malformations has contributed to fetal outcome:A 22 year review. Fetal Diagn Ther 1993;8(6):388-401.

47. Smithells RW, Sheppard S. Possible prevention of neural-tube defects by periconceptional vitamin supplementation. Lancet 1980 Mar 22; I(8169):647.

48. Tolarova M. Periconceptional supplementation with vitamins and folic acid to prevent recurrence of cleft lip. Lancet 1982 Jul 24;2(8291):217.

49. C zeizel AE, D udas I. Prevention of the first occurrence of neural-tube defects by periconceptional vitamin supplementation. $\mathrm{N}$ Engl J Med 1992;327:1832-1835.

50. Sean D, Mills JL, Malloy Am, Conley M, Lee YJ, Kirke PN et al. Minimum effective dose of folic acid for the food fortification to prevent the tube neural defects. Lancet 1997 Dec 6:350(9092):1666-1669. 